
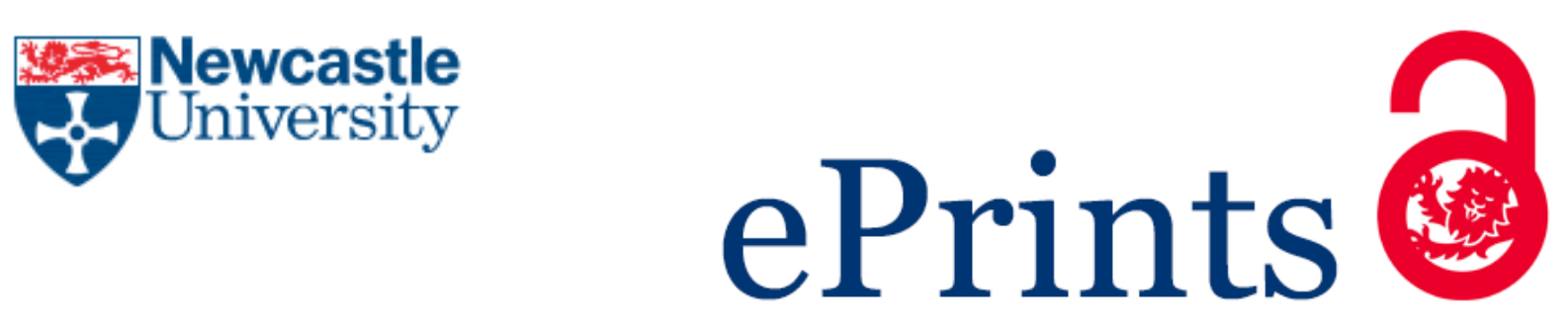

Taylor JJ, Preshaw PM. Gingival crevicular fluid and saliva. Periodontology $20002016,70(1), 7-10$.

\title{
Copyright:
}

This is the peer reviewed version of the following article: Taylor JJ, Preshaw PM. Gingival crevicular fluid and saliva. Periodontology 2000 2016, 70(1), 7-10. which has been published in final form at http://dx.doi.org/10.1111/prd.12118 This article may be used for non-commercial purposes in accordance with Wiley Terms and Conditions for Self-Archiving.

Date deposited:

$12 / 01 / 2016$

Embargo release date:

10 December 2016

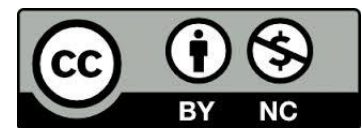

This work is licensed under a Creative Commons Attribution-NonCommercial 3.0 Unported License 
Gingival crevicular fluid and saliva

John J. Taylor \& Philip M. Preshaw

John J Taylor PhD

Centre for Oral Health Research \&

Institute of Cellular Medicine

Level 7

School of Dental Sciences

Newcastle University

Framlington Place NE2 4BW UK

Tel: +44(0)1912088694 (Office)

Tel: +44(0)191208 6137 (Fax)

e-mail: john.taylor@ncl.ac.uk

Running title: GCF and saliva

Monograph title: Gingival crevicular fluid and Saliva

(Guest Editors: John J. Taylor \& Philip M. Preshaw) 


\section{Gingival crevicular fluid and saliva}

\section{Introduction}

Understanding the structure and function of the mouth, its tissues and secretions is of great interest to physiologists, cell biologists, immunologists and microbiologists but is also of fundamental interest to the dental professional interested in comprehending the aberrant processes associated with oral disease and in the application of effective clinical interventions. The field of periodontology, which has a truly multidisciplinary perspective cutting across leading edge molecular and cellular biology, clinical dentistry, epidemiology and behavioural science, exemplifies this. A paradigm shift in recent years has led to the consideration of the oral cavity (and, thus, oral disease) not in isolation but as a component integrated with systemic physiology, important in maintaining systemic health and reflective of systemic disease; this has served to promote periodontology, in particular, into the forefront of medicine in general (2). This volume considers the role of gingival crevicular fluid (GCF) and saliva in physiological function, maintenance of oral tissue integrity, defense against pathogens and oral disease as well as the many, emerging applications of analysis of these fluids in support of periodontal disease diagnosis, prognosis and epidemiology. However, whilst the emphasis is on periodontal disease, the wider contexts of oral and systemic health are also key considerations in this volume.

\section{Physiological regulation of saliva secretion}

The oral cavity is a unique anatomical structure, characterised by the juxtaposition of soft and hard tissues and which is continuously subject to challenge by the external environment and foreign material. The mouth is well served by numerous major and minor salivary glands and the saliva they secrete is critical for physiological function and a key component of the host defence against infection in the mouth. Proctor (14) describes the structure and function of the major and minor salivary glands and their secretory processes. Understanding the physiological and pharmacological regulation of salivary secretion and its variation in health and disease are critical to 
the clinical management of compromised salivary secretion (xerostomia) which often underpins episodes of caries, periodontitis and candidiasis in patients with other disorders.

\section{Salivary biomarkers for periodontal disease}

Korte and Kinney (11) outline the use of saliva as a source of biomarkers for periodontal disease diagnosis and monitoring. Analysis of expectorated saliva (oral fluid) is gaining popularity, as it is relatively straightforward to collect and contains a large proportion of the total gingival crevicular fluid from the periodontal pockets in the whole mouth. Furthermore, it is clear that analysis of individual elements of the pathogenesis of complex and variable diseases such as periodontitis is not as useful (or desirable) as the investigation of multiple elements that provide overlapping but distinct disease associations. Korte and Kinney describe the identification of host response and microbiological markers for periodontitis and their efficacy in periodontal disease management.

\section{'Omics' and salivary biomarker discovery}

Our limited understanding of the molecular details of disease pathogenesis restricts identification of candidate biomarkers for periodontitis. Also, numerous mediators with clearly defined pathogenic roles in in vitro studies have not proven to have good quantitative disease associations. Zhang et al (17) describe unbiased approaches to biomarker discovery using global analysis of RNA and proteins in saliva which have identified novel biomarkers and are beginning to yield valuable information about the molecular biology of oral diseases. The application of this apporach is clearly not limited to peridontitis and the authors outline applications to biomarker discovery for wider oral and systemic disease diagnosis and management.

\section{Gingival crevicular fluid function and analysis}

The connective tissues of the periodontium are highly vascular, facilitating emigration of the molecular and cellular components of blood into the periodontal tissues; thus the gingival sulcus is bathed with gingival crevicular fluid. Gingival 
crevicular fluid is a serum exudate that carries all the key molecular (complement components, antibodies) and cellular (neutrophils and plasma cells) components of the immune response necessary to prevent tissue invasion by the subgingival plaque bacteria. Collection and analysis of GCF has long been a popular approach to investigating localised inflammatory processes in periodontitis; Barros et al (1) describe the formation and dynamics of gingival crevicular fluid as well as exploring the application of gingival crevicular fluid analysis as a source of biomarkers for periodontal disease. Barros et al empahsise the potential of the metabolomic analysis of gingival crevicular fluid as a potential approach to develop biomarkers which more accurately reflect host-pathogen interactions.

In a complementary article, Wassell and Preshaw (16) outline the clinical and technical challenges involved in the analysis of this fluid in studies of periodontitis and the barriers to accurate interpretation of the many clinical studies of gingival crevicular fluid in the periodontal literature. It is almost a platitude in clinical translational research that differences in findings might be explained by differences in methodology; Wassall and Preshaw clearly set out fundamental requirements of study design and reporting if we desire to produce studies comparable with other independent studies in the future.

\section{Influence of saliva on the oral microbiota and infectious disease}

The oral cavity is a unique anatomical structure, characterised by the juxtaposition of soft and hard tissues and which is continuously subject to challenge by the external environment and foreign material. Diseases and disorders caused by oral microorganisms such as periodontitis and dental caries are very common and economically important. Also, the mouth is a site for systemic infection and its tissues and secretions are a repository for infective pathogens that have entered the body elsewhere. It is increasingly apparent that control of host/microbiome interactions in the soft and hard tissues of the mouth is central to the maintenance of oral health; collapse of the immune response, for example, in acquired immunodeficiency syndrome leads to the rapid onset of severe oral pathologies mostly driven by infectious agents. The saliva has many molecular elements that modulate pathogenic microorganisms. Marsh et al (12) describe the salivary factors 
that influence the development of oral biofilms and outline the protective role of saliva on the pathogenesis of oral disease. Meanwhile, Corstjens and colleagues (3) outline the application of the analysis of saliva in the diagnosis and investigation of viral diseases. The role of saliva in studies of HIV and the interaction of salivary proteins with HIV is highlighted. Hassona and Scully (9) explain the causes and clinical consequences of salivary hypofunction emphasising the role of saliva in protection against oral mucosal diseases including candidiasis, burning mouth syndrome and lichen planus.

\section{Application of biomarkers to monitor caries and periodontal disease}

Gao et al (6) highlight the role of saliva in the development of dental caries and review current information on salivary biomarkers in dental caries. These authors highlight the combined effect of the complex biochemical elements of saliva in indirectly (via the formation of acquired pellicle) and directly (through many antimicrobial actions) protecting against mutans streptococci and other potentially cariogenic bacteria. This article also explores the potential for salivary analysis in revealing biomarkers of dental caries but at the same time reminds us of the importance of holistic consideration of caries susceptibility factors including environmental exposures, socio-demographic factors and behavioural variables in assessing caries risk.

It has long been recognised that inappropriate regulation of host matrix metalloproteinases and neutrophil collagensase (matrix metalloproteinase-8) in particular is central to the destructive processes associated with periodontitis. Indeed matrix metalloproteinases are bona fide therapeutic targets in periodontal disease management (13). Thus, matrix metalloproteinases have been the subject of intense study and Sorsa et al (15) review our knowledge of salivary and gingival crevicular fluid matrix metalloproteinases as biomarkers for oral diseases and highlight the application of matrix metalloproteinases analysis in the management of periodontitis and peri-implantitis.

Cytokines orchestrate immune response in tissues and mediate inflammation. An emerging principle is the appreciation of the wider role of cytokines and molecules with cytokine-like activity (e.g. adipokines) in other 
physiological processes such as development, tissue homeostasis and repair. Jaedicke et al (10) explain the relationship of salivary cytokines to periodontal disease severity and progression, and assess the utility of salivary cytokines, and related molecules, as markers of periodontal disease. Despite our considerable knowledge of the role of cytokines in inflammatory disease pathogenesis, few of these molecules can yet be described as bona fide biomarkers for periodontitis. Holistic analysis of host immune responses and improved clinical study design may, however, reveal new markers.

\section{Saliva in studies of epidemiology of human disease}

Saliva collection is a simple, painless procedure not requiring skilled personnel; therefore if saliva collection can be coupled with suitable analytical protocols, salivary analysis has great potential in large clinical trials, in self-management of disease and in epidemiological studies in regulated clinical environments but also in the field, in locations where access to clinical facilities is scarce. Galloway et al (5) describe the role of saliva collection and analysis in prospective, population-based studies of human disease. Potential applications of saliva in the investigation of human disease and disorder in large, national cohort studies where the results of salivary analysis can be compared to comprehensive medical records, lifestyle factors and other genetic and biochemical data are also explained.

\section{Conclusion}

This volume has addressed the function of elements of gingival crevicular fluid and saliva, how they interact with host cells and pathogens, and describes how the application of biomarkers within these oral fluids studies might have oral health benefits. It is critical that this field should be underpinned by strong microbiology (to understand complexity of oral microbiome) and cell biology/immunology to understand host defences, tissue repair and regeneration. Clear exposition of methodological approaches, careful selection and calibration of analytical tools and appropriate experimental design and analysis are also essential. A clear theme of these reviews is the need for a holistic framework in which to integrate this biological information. A number of challenges are ahead: we still do not completely 
understand how confounding factors such as smoking and systemic diseases (e.g. diabetes) impinge on the biochemical and cellular components of oral fluids and hence their utility in periodontal diagnosis and monitoring. Once we can embrace these complexities and overcome the technical challenges, then analysis of oral fluids could significantly enhance oral disease management in the clinic and (potentially) the home. Saliva sampling is particularly efficacious and acceptable to patients and clinicians alike $(7,8)$ and this coupled with developments in chair side diagnostic platforms (4) has the potential the herald a new era in oral and periodontal healthcare. 


\section{References}

1. Barros SP, Williams R, Offenbacher S, Morelli T. GCF as a source of biomarkers for periodontitis. Periodontol 2000 2015:

2. Chapple IL. Time to take periodontitis seriously. B Med J 2014: 348: g2645

3. Corstjens PLAM, Abrams WR, Malamud D. Saliva and viral infections. Periodontol 2000 2015:

4. Fuentes L, Yakob M, Wong DT. Emerging horizons of salivary diagnostics for periodontal disease. Br Dent J 2014: 217: 567-573

5. Galloway JW, Keijser BJF, Williams DM. Saliva in studies of epidemiology of human disease. Periodontol 2000 2015:

6. Gao X, Jiang S, Koh D, Hsu C-YS. Salivary biomarkers for dental caries. Periodontol 2000 2015:

7. Greenberg BL, Glick M, Julie F-H, Kantor ML. Dentists' attitudes toward chairside screening for medical conditions. J Am Dent Assoc 2010: 141: 5262.

8. Greenberg BL, Kantor ML, Jiang SS, Glick M. Patients' attitudes toward screening for medical conditions ion a dental setting J Pub Health Dent 2012: 72: 28-35.

9. Hassona Y, Scully C. Salivary changes in oral mucosal diseases. Periodontol 2000 2015:

10. Jaedicke KM, Preshaw PM, Taylor JJ. Salivary cytokines as biomarkers of periodontal disease. Periodontol 2000 2015:

11. Korte DL, Kinney J. Personalized medicine: An update of salivary biomarkers for periodontal diseases. Periodontol 2000 2015:

12. Marsh PD, Do T, Beighton D, Devine DA. Influence of saliva on the oral microbiota. Periodontol 2000 2015:

13. Preshaw PM. Host response modulation in periodontics. Periodontology 2000 2008: 468: $92-110$

14. Proctor G. The physiological regulation of saliva secretion. Periodontol 2000 2015: 
15. Sorsa T, Gursoy UK, Nwator S, Hernandez M, Tervahartiala T, Leppilahti J, Gursoy M, Könönen E, Emingil G, Pussinen PJ, Mäntylä P. Analysis of matrix metalloproteinases in gingival crevicular fluid (GCF), mouthrinse and saliva for monitoring periodontal diseases. Periodontol 2000 2015:

16. Wassall, RR, Preshaw PM. Clinical and Technical considerations in the analysis of GCF. Periodontol 2000 2015:

17. Zhang $Y$, Sun J, Lin C-C, Abemayor E, Wang MB, Wong DTW. The emerging landscape of salivary diagnostics. Periodontol 2000 2015: 